\title{
Enhanced cognitive-behavioral therapy for vocational rehabilitation in schizophrenia: Effects on hope and work
}

\author{
Paul H. Lysaker, PhD; ${ }^{1-2 *}$ Gary Bond, PhD; ${ }^{3}$ Louanne W. Davis, PsyD; ${ }^{1}$ Gary J. Bryson, PsyD; ${ }^{4-5}$ \\ Morris D. Bell, $\mathbf{P h D}^{4-5}$ \\ ${ }^{1}$ Roudebush Department of Veterans Affairs (VA) Medical Center, Indianapolis, IN; ${ }^{2}$ Indiana University School of \\ Medicine, Indianapolis, IN; ${ }^{3}$ Indiana University-Purdue University, Indianapolis, IN; ${ }^{4}$ VA Health Care Connecticut, \\ West Haven, CT; ${ }^{5}$ Yale University School of Medicine, New Haven, CT
}

\begin{abstract}
To address the effects of dysfunctional cognitions on vocational outcome of people with schizophrenia spectrum disorders, we developed the Indianapolis Vocational Intervention Program (IVIP), a cognitive-behavioral program of group and individual interventions. Fifty participants with schizophrenia or schizoaffective disorder were offered 6-month work placements and randomized to receive IVIP $(n=25)$ or standard support services $(n=25)$. Hours worked were measured weekly, and work performance was assessed biweekly with the use of the Work Behavior Inventory. Hope and self-esteem were assessed at baseline and at 5 months with the Beck Hopelessness Scale and the Rosenberg Self-Esteem Schedule. Analysis of variance (ANOVA) revealed that the IVIP group worked significantly more weeks and had better average work performance than the standard support group. Repeated measures ANOVA of baseline and follow-up scores indicated that the IVIP group sustained baseline levels of hope and self-esteem through follow-up, while the standard support group experienced declines. Results provide initial evidence of the effectiveness of the IVIP.
\end{abstract}

Key words: cognitive-behavioral therapy, dysfunctional beliefs, hope, psychosocial rehabilitation, schizophrenia, self-efficacy, self-esteem, severe mental illness, vocational function, work.

\section{INTRODUCTION}

Many unemployed or disabled adults with schizophrenia spectrum disorders wish to work again yet doubt their ability to succeed [1-3]. As the result of factors including stigma [4-5], practitioners' negative expectations, and the deficits associated with severe mental illness, many with schizophrenia spectrum disorders view themselves as being minimally competent, of low social value, and possibly beyond help [6-8]. They may believe that they have little ability to influence their lives [9-12] and construct a personal narrative in which they expect social and vocational failure [13]. Thus these beliefs not only are distressing but also may serve as self-fulfilling

Abbreviations: ANOVA $=$ analysis of variance; $\mathrm{BHS}=$ Beck Hopelessness Scale; CBT = cognitive-behavioral therapy; DSM-IV ${ }^{\circledR}=$ Diagnostic and Statistical Manual of Mental Disorders, Fourth Edition; ICC = intraclass correlation coefficient; IVIP = Indianapolis Vocational Intervention Program; PANSS = Positive and Negative Syndrome Scale; RSES = Rosenberg SelfEsteem Schedule; SCID-I = Structured Clinical Interview for the DSM-IV $^{\circledR}$ Axis I Disorders; SD = standard deviation; VA = Department of Veterans Affairs; WBI = Work Behavior Inventory. This material was based on work supported by the VA Rehabilitation Research and Development Service, grant D-2570R.

*Address correspondence to Paul H. Lysaker, PhD; Day Hospital 116H, 1481 West 10th Street, Roudebush VA Medical Center, Indianapolis, IN 46202; 317-554-0000, ext. 2546; fax: 317-554-0056. Email: plysaker@iupui.edu

DOI: 10.1682/JRRD.2004.12.0157 
prophecies that limit function independent of other social or biological factors. For instance, given a belief that insurmountable difficulties are destined to occur at work, many persons may conclude that persevering is pointless when problems arise on the job. Therefore, they might stop problem-solving and give up. Some may continue for a while, but their negative expectations cause them to interpret most events, even positive ones, as evidence of impending failure.

Consistent with these assertions and studies of persons without severe mental illness [14], negative beliefs about self-efficacy predict poorer employment outcome in persons with schizophrenia spectrum disorders [15-17]. Even after controlling for negative symptoms, one prospective study found that when participants were faced with difficulties, their dysfunctional beliefs about giving up significantly predicted poor rehabilitation outcome [8]. Negative beliefs about the self have also been linked to other behaviors that may compromise vocational function, including avoidant coping $[9,18]$, poor participation in treatment [19], and generally poor social function [7,20].

One interpretation of the literature in this area is that, beyond needing more opportunities and concrete skills, persons with schizophrenia need new ways of thinking about themselves as workers. Indeed, many of the most sophisticated evidence-based vocational programs, which provide opportunity and support, find disappointingly short job tenures [21]. To address these challenges, we have developed a cognitive-behavioral group and individual treatment program called the Indianapolis Vocational Intervention Program (IVIP). As described elsewhere [22], we developed the IVIP manual while working with 20 participants with diagnoses of schizophrenia or schizoaffective disorder as confirmed by the Structured Clinical Interview for the Diagnostic and Statistical Manual of Mental Disorders, Fourth Edition (DSM-IV ${ }^{\circledR}$ ) Axis I Disorders (SCID-I) [23]. Participants in a postacute phase of their illness were recruited from outpatient clinics at a Department of Veterans Affairs (VA) medical center.

In designing this intervention, we applied and adapted a wide range of existing cognitive-behavioral therapy (CBT) didactic materials and techniques to target dysfunctional beliefs that might affect vocational function. We chose to use CBT principles to address dysfunctional beliefs because CBT helps alter a wide range of cognitions. More importantly, research over the last decade has indicated that CBT helps persons with schizophrenia spectrum disorders reduce positive and negative symptoms as well as increase community tenure [24-28].

The IVIP offers weekly group and individual CBT sessions targeted at dysfunctional beliefs about self (e.g., "I cannot succeed") and work experiences (e.g., "My supervisor criticizes my work and dislikes me"). The IVIP group therapy sessions are provided for 1 hour a week and involve three essential activities:

1. Teaching the week's didactic material.

2. Helping participants practice the didactic material with an application exercise.

3. Giving work feedback to participants.

The didactic material is organized into four 2-week modules (total of eight sessions) that are presented in order and repeated at least three times during the 6-month program. The four modules are "Thinking and Work," "Barriers to Work," "Workplace Relationships," and "Realistic Self-Appraisal." The IVIP individual sessions are also weekly and complement the group sessions by offering more in-depth and personalized application of group material to beliefs participants hold about themselves and their work experiences. Participants also have the opportunity in individual sessions to use a running record to identify and explore dysfunctional beliefs. The IVIP is intended as an adjunct to work therapy programs, including those that provide paid work placements to participants. Such programs include Incentive Therapy, Veterans Industries, and Compensated Work Therapy [29], which are available in more than 109 VA sites across the country.

While we have reported cases illustrating many participants' ready acceptance of the IVIP [22], whether this program improves dysfunctional cognitions, work performance, and possibly psychiatric symptoms is unclear. This article presents a randomized feasibility study of the IVIP that tested whether participants receiving IVIP along with their work placement would demonstrate higher levels of hope and self-esteem, better vocational function, and lower levels of psychiatric symptoms than participants receiving a work placement with standard support services.

\section{METHODS}

\section{Design}

This study was a 26-week, randomized controlled trial of the IVIP. Symptoms, self-esteem, and hope were 
measured at baseline and then again at 5 months. Hours of work were collected weekly and work performance was assessed biweekly.

\section{Participants}

Fifty males with SCID-I-confirmed diagnoses of schizophrenia $(n=37)$ or schizoaffective disorder $(n=13)$ were recruited from a VA outpatient psychiatry service, where all were receiving medication management by an assigned clinician. On average, participants were 48.1 years old \pm 5.7 standard deviation (SD), had 12.5 years of education $\pm 1.2 \mathrm{SD}$, and 10.5 lifetime psychiatric hospitalizations \pm 9.52 SD with the first occurring at the age of $24.7 \pm 6.2 \mathrm{SD}$. All participants were in a postacute phase of illness as defined by no hospitalizations or changes in psychotropic medication or housing in the month before entering the study. Participants who had a diagnosis of mental retardation or another neurological disorder were excluded. Twenty-eight participants were African American, twenty-one Caucasian, and one Latino.

All participants had been unemployed for a minimum of 2 years and were referred to us by their clinician. Most participants had been receiving psychiatric services at this VA for more than 2 years. Eighteen (ten in the IVIP group and eight in the standard support group) had received some previous form of vocational rehabilitation service at the VA. None received concurrent vocational services while participating in the research program.

\section{Instruments}

The Beck Hopelessness Scale (BHS) is a 20-item questionnaire that asks participants to endorse statements about expectations of success or failure as true or false [30]. Item responses are then summed to provide a total score such that a higher score indicates more hope. Examples of items include "Things just won't work out the way I want them to" and "I might as well give up because I can't make things better for myself.” This scale has been used successfully with a wide range of psychiatric, medical, and community populations [16,31-32]. Evidence of acceptable internal consistency and convergent validity has been reported elsewhere [33-34].

The Rosenberg Self-Esteem Schedule (RSES) is a 10-item questionnaire that asks participants to indicate the degree of their agreement or disagreement with statements about their self-esteem and self-deprecation [35]. Item responses are summed into a total score such that a higher score indicates greater self-esteem. Examples of items include "I wish I could have more respect for myself" and "I feel that I have a number of good qualities." The RSES has been widely used in community samples, and literature suggests that persons with schizophrenia spectrum disorders can also reliably complete this questionnaire [36]. Reliability coefficients from previous studies of clients with severe mental illness found internal consistency coefficients (Cronbach's $\alpha$ ) exceeding 0.80 and test-retest reliability of 0.87 [37].

The Positive and Negative Syndrome Scale (PANSS) is a 30-item rating scale that clinically trained research staff complete following a chart review and semistructured interview with the participant [38-39]. Interrater reliability for raters in this study was good to excellent on all scale scores, with intraclass correlation coefficients (ICCs) ranging from 0.82 to 0.93 . PANSS raters in this study were aware of participant assignment to condition.

The Work Behavior Inventory (WBI) is a 35-item inventory that specifically assesses the work behavior of persons with severe mental illness [40]. A trained rater completes the WBI following direct observation of the participant's work behavior and an interview with the participant's supervisor. Each WBI item consists of a specific behavior that is rated as a 1 (persistent problem area), 2 (occasional problem area), 3 (average performance), 4 (occasional area of strength), or 5 (frequent area of strength). Items are used to generate a total score that is the sum of five subscales: social skills, cooperativeness, work habits, work quality, and personal presentation. Data supporting the factorial and concurrent validity of the WBI have been reported elsewhere [40]. Interrater reliability was good to excellent for raters in this study, with ICCs ranging from 0.82 to 0.94 . WBI scores have also been found to predict subsequent vocational outcomes [41]. WBI raters in this study were aware of participant assignment to condition.

\section{Procedures}

After obtaining the participant's written informed consent, a research psychologist or trained research assistant with bachelor's or master's level training in psychology administered baseline assessments of the BHS, RSES, and PANSS. He or she was available to read or discuss items with participants who had difficulty. Following the completion of baseline assessment, participants were assigned by block randomization to receive either the IVIP or standard support services. All participants received offers of and accepted 26-week work placements 
in entry-level medical center positions supervised by the regular work-site supervisors. Participants were paid $\$ 3.50$ an hour for up to 20 hours a week of work activity. While all participants were guaranteed a placement, they could be terminated for failure to follow the rules of the work site or for substandard performance as determined by the work-site supervisor.

Services provided in the IVIP are described in detail elsewhere [22], and the IVIP manual is available from the authors. The overarching purpose of the IVIP is to help participants identify and correct dysfunctional beliefs about work. Support services in the standard support condition were modeled after VA work programs and included a weekly group that lasted up to an hour and offered support and discussion of work-related issues and concerns. Unlike the IVIP group sessions, the standard support group sessions contained no curriculum, gave no specific work feedback, and relied upon material brought up for discussion by the participants. The therapist who provided the standard support services was not the same therapist who provided the IVIP group and individual interventions.

Participants worked regularly scheduled hours at work sites, such as the escort service, which included helping patients in wheelchairs travel through the hospital to appointments; housekeeping, which included performing general janitorial and laundry work; and medical administration, which included filing, answering phones, and informing practitioners when persons with scheduled appointments arrived. Work duties were equivalent to entry-level positions, and hospital staff supervisors provided supervision. Participants were expected to work between 10 and 20 hours a week as determined by their own wishes. Efforts were made to match work placements with participants' interests and skills. Work behavior was evaluated biweekly with the WBI. Participants recorded their hours worked on weekly time cards that were verified and signed by their work supervisor. To assess changes in cognitions and symptoms, study personnel readministered the BHS, RSES, and PANSS 5 months later. Of note, while the program lasted 6 months, 5 months was chosen as the follow-up point. Five months was deemed sufficient time for the intervention to take effect but avoid additional unwanted variance introduced by participants experiencing demoralization at the conclusion of the program, as noted in past research. While many participants were acquainted with one another, we did not prevent IVIP participants from sharing their treatment experiences with standard support participants.

\section{Analysis}

Of the initial 50 participants, 3 were not included in the final analyses. From the IVIP group, one participant left the state and was unavailable for follow-up and a second participant became severely disorganized immediately following the baseline assessments and was hospitalized in a long-term facility an hour away. To date, he has not been discharged. From the standard support group, one participant was excluded because he was able to obtain individual CBT on his own.

We compared outcomes between groups in two steps. First, we examined work-related behavior. Group means for total hours worked and weeks of work over the 6 months of active intervention were compared using analysis of variance (ANOVA). Two participants in the standard support group did not work long enough for WBI evaluations, although they completed the other measures. One participant in the standard support group who worked long enough for WBI evaluations declined the symptom interviews and refused to complete the hopelessness and self-esteem measures. In the second step, we used three separate repeated measures ANOVAs to compare groups on hope, self-esteem, and symptoms from baseline to 5-month follow-up. All comparisons were two-tailed with $\alpha=0.05$.

\section{RESULTS}

Group comparisons revealed no differences in age, education, hospitalization history, or diagnosis between participants randomized to receive IVIP versus standard support services (Table 1). Groups did not differ at baseline on the PANSS, BHS, or RSES. A $t$-test comparing the number of groups attended indicated that participants in the IVIP group attended significantly more groups (16.4 $\pm 7.53 \mathrm{SD}$ ) than participants in the standard support group (11.5 $\pm 10.09 \mathrm{SD} ; t=2.18, p<0.05)$. Over the 6 months, the average IVIP participant also attended 69 percent (18.4 \pm 7.56 SD) of scheduled individual sessions. Two participants in both groups were dismissed from their placements but are included in all analyses.

As presented in Table 2, ANOVAs comparing work outcomes revealed that participants in the IVIP group worked significantly more weeks than those in the standard support group. Differences in the total number of hours worked between groups approached but did not achieve statistical significance. WBI total scores were 
Table 1.

Participant $(N=50)$ background characteristics (mean \pm standard deviation).

\begin{tabular}{lcc}
\hline \multicolumn{1}{c}{ Characteristic } & IVIP & Standard Support \\
\hline Age (yr) & $46.42 \pm 6.97$ & $49.68 \pm 4.90$ \\
Education (yr) & $12.38 \pm 1.01$ & $12.68 \pm 1.43$ \\
No. Lifetime Psychiatric & $10.63 \pm 9.97$ & $9.96 \pm 9.31$ \\
$\quad$ Hospitalizations & & \\
Age at First Psychiatric & $26.13 \pm 6.97$ & $23.29 \pm 9.31$ \\
$\quad$ Hospitalizations (yr) & 17 & 20 \\
Schizophrenia (No.) & 8 & 5 \\
Schizoaffective (No.) & & \\
\hline
\end{tabular}

averaged for each individual across weeks of work to produce an individual mean score. WBI total scores were significantly greater for the IVIP group than for the standard support group, although this finding may have been influenced by raters' knowledge of participant assignment to condition.

As seen in Table 3, repeated measures ANOVAs comparing baseline and follow-up scores for the BHS, RSES, and PANSS revealed significant group, time, and interaction effects for the BHS and RSES scores. Multiple comparisons of individual scores revealed that the IVIP group had significantly higher scores on the BHS and RSES than the standard support group at follow-up $(\mathrm{p}<0.01)$ but not at baseline. Further analysis revealed that these differences at follow-up were the result of the significant BHS and RSES declines from baseline to 5 -month follow-up for the standard support group $(p<0.01)$. The BHS and RSES levels for the IVIP group as a whole were stable and did not differ statistically from baseline to follow-up. No significant effects or interactions were found for the PANSS, although a trend suggested that the sample as a whole showed symptomatic improvement from baseline to follow-up ( $p<0.10)$. Again, raters' awareness of participant assignment to condition may have influenced results.

To examine whether levels of hope at follow-up were linked to work outcomes independent of baseline, we used Pearson's correlation ( $r$ ) to partially correlate BHS total score at follow-up with weeks of work, hours of work, and WBI total score, controlling for BHS total score at baseline. These analyses revealed that BHS total score at follow-up was positively correlated with more weeks of work $(r=0.33, p<0.05)$ but not with WBI total score or hours of work. Finally, to examine whether levels of self-esteem at follow-up were linked to work outcomes independent of baseline, we partially correlated RSES total score at follow-up with weeks of work, hours of work, and WBI total score, controlling for RSES total score at baseline. These analyses revealed that RSES level at follow-up was positively linked at the trend with more weeks of work $(r=0.28, p=0.08)$ and hours of work ( $r=0.29, p=0.06)$ but not with WBI total score.

\section{DISCUSSION}

The IVIP was created to promote vocational function by correction of dysfunctional patterns of work-relevant thought and behavior. Results provide preliminary support for the efficacy of the program. Participants with a significant history of dysfunction, including multiple hospitalizations and long periods of unemployment, showed greater adherence to the IVIP by participating in more than 66 percent of scheduled counseling appointments, as compared with participants in the standard support group who attended 42 percent of their scheduled visits. IVIP participants worked more weeks, achieved better average

Table 2.

Analyses of variance comparing work outcomes between IVIP $(n=23)$ and standard support $(n=24)$ groups.

\begin{tabular}{lcccc}
\hline \multicolumn{1}{c}{ Measure } & $\begin{array}{c}\text { IVIP } \\
\text { (Mean } \pm \text { SD) }\end{array}$ & $\begin{array}{c}\text { Standard Support } \\
\text { (Mean } \pm \text { SD) }\end{array}$ & F & $\boldsymbol{p}^{-V a l u e}$ \\
\hline Weeks of Work & $20.39 \pm 8.00$ & $13.71 \pm 10.44$ & 6.03 & 0.02 \\
Hours of Work & $347.10 \pm 158.20$ & $243.88 \pm 120.61$ & 3.66 & 0.06 \\
Mean WBI Total $^{\dagger}$ & $118.34 \pm 20.30$ & $107.55 \pm 8.49$ & 5.12 & 0.03 \\
${ }^{*} p<0.05$ is significant. & & \\
${ }^{\dagger}$ Two participants in support group were dropped from this comparison because they did not work enough hours for performance to be assessed. \\
$F=$ variance ratio score, IVIP = Indianapolis Vocational Intervention Program, SD = standard deviation, WBI = Work Behavior Inventory. \\
\hline \hline
\end{tabular}


work performance, and maintained baseline levels of hope for the future and better self-esteem over 6 months at paid work placements than participants receiving standard support services. Contrary to expectations, groups did not differ on symptom levels at follow-up. Previous studies have indicated that work programs can directly yield symptom improvement and that any effects of CBT may come indirectly through enhancement of vocational function. If so, the relatively small sample size of this study would not provide enough statistical power to reveal such indirect effects. Thus, our findings do not provide evidence that CBT affects symptoms. If symptom improvement is to be a direct goal, the IVIP may need to include curricula aimed specifically at reducing symptoms.

The IVIP and standard support groups differed in hours worked only at the trend level. This may be the result of our small sample size and one should note that a medium effect size was found for this variable. The effectiveness of the IVIP in helping persons persist at work may possibly be reflected in significantly more weeks worked rather than sheer amount of hours spent on the job. As noted in the introduction, many persons with schizophrenia spectrum disorders are hypothesized to leave work when they expect nothing is to be gained from perseverance. Put another way, we speculate that quitting a job may be more sensible to the individual than staying if failure and humiliation seem likely. We hypothesize that the IVIP participants kept trying because they learned to question their automatic thoughts and dysfunctional beliefs and thereby sustained hopes of success. A longer period of observation (for example, 12 months) might show that increased persistence (more weeks worked) eventually produces more hours of work activity.

The pattern of hope and self-esteem scores for the two groups also suggests hypotheses for future research. While the IVIP group was able to sustain baseline levels of hope and self-esteem (although we expected these levels to improve), the standard support group declined from baseline levels. One interpretation that could be tested in future studies is that both groups came to work with considerable enthusiasm. In preparing to work after several years of unemployment, many participants may have tried to bolster themselves by thinking about positive outcomes. The IVIP participants, though, were the ones who maintained their levels of hope and self-esteem as a group. Possibly, participants in the standard support group found work demoralizing and were more likely to quit. In contrast, the participants in the IVIP group may have been less demoralized by frustrating work experiences because they were able to problem-solve and avoid making negative self-attributions. Of note, the standard support condition was modeled after prevailing support services and whether such services actually help people is unclear. The current study, at a minimum, suggests that unstructured support does not sufficiently curb discouragement and negative beliefs. Whether such support groups actually have negative effects is uncertain but, in any case, one could reasonably conclude that a structured curriculum emphasizing hope and recovery will be more effective than one with no structure.

Table 3.

Repeated measures analyses of variance comparing total scores between IVIP $(n=23)$ and standard support $(n=24)$ groups on measures of hope (BHS), self-esteem (RSES), and symptoms (PANSS) from baseline to 5-month follow-up.

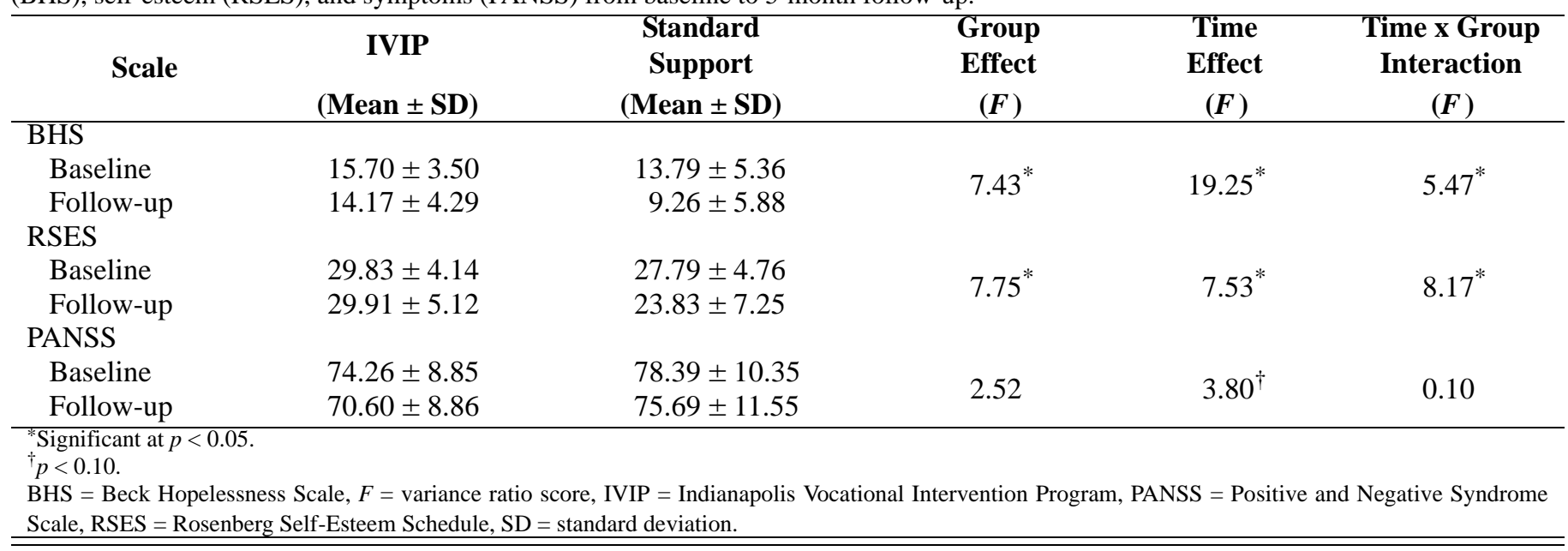


Rival interpretations of our results cannot be ruled out. First, in keeping with the standard services available, the standard support group did not receive individual counseling; therefore, these participants had fewer hours of professional contact each week. Thus, all effects were possibly the result of the IVIP participants receiving twice as many hours of service or simply provider contact each week. Also possible is that the IVIP was merely a more compliant group and thus more likely to adhere to the expectations of the work sites. Rater bias is another factor that could have affected work performance assessments, since raters were aware of assignment to condition. Many intervening variables were not assessed that could have contributed to gains in vocational function and the stability of hope and self-esteem, including internalized stigma (when persons with schizophrenia are biased against themselves because they have mental illness). Thus, findings of this study should be considered tentative and a basis for future study before much weight is accorded them.

This study has several important limitations. Participants were males generally in their forties enrolled in treatment. Thus, how well the findings generalize to women, persons early in their illness, and persons who decline services is unknown. Additionally, participants in this report were placed in temporary entry-level jobs in a VA medical center in keeping with participants' interests. As numerous programs throughout the VA system provide placements identical to those we have used, our program is potentially exportable to them. Therefore, these placements appear to be valid objects of study because they represent actual work experiences that can be a part of persons' vocational aspirations, even though the participants do not become permanent "employees." Unknown, however, are whether and what modifications are necessary to apply the IVIP in other vocational settings, including those that provide supported employment.

\section{CONCLUSION}

Use of CBT as a treatment strategy for persons with schizophrenia spectrum disorders is a relatively new endeavor, and ours is one of the first attempts to apply CBT specifically to enhance vocational function. We have developed a manualized treatment with measures of fidelity so that replication studies can be conducted to further test its efficacy. Because the IVIP has many ele- ments, more study will be needed to determine which element is most important or whether the program is greater than the sum of its parts. In future studies, we also plan to use raters who are unaware of the experimental conditions to which participants are assigned, include a more diverse group of persons with schizophrenia spectrum disorders, and assess other intervening variables such as internalized stigma. Furthermore, we plan to use a control group that receives an equal amount of service and to assess the extent to which control participants use CBT principles. Finally, we will to explore the effects of the intervention when it is provided for differing lengths of time.

\section{REFERENCES}

1. Bebout RR, Harris M. Personal myths about work and mental illness: response to Lysaker and Bell. Psychiatry. 1995;58(4):401-4.

2. Roe D. Progressing from patienthood to personhood across the multidimensional outcomes in schizophrenia and related disorders. J Nerv Ment Dis. 2001;189(10):691-99.

3. Young SL, Ensign DS. Exploring recovery from the perspective of persons with psychiatric disabilities. Psychosoc Rehabil J. 1999;22:219-31.

4. Phelan JC, Link BG, Stueve A, Pescosolido BA. Public conceptions of mental illness in 1950 and 1996: what is mental illness and is it to be feared? J Health Soc Behav. 2000;41(2):188-207.

5. Martin JK, Pescosolido BA, Tuch SA. Of fear and loathing: the role of 'disturbing behavior,' labels, and causal attributions in shaping public attitudes toward people with mental illness. J Health Soc Behav. 2000;41(2):208-23.

6. Hays JR, Buckle KE. Self-efficacy among hospitalized mentally ill patients. Psychol Rep. 1992;70(1):57-58.

7. Roe D. A prospective study on the relationship between self-esteem and functioning during the first year after being hospitalized for psychosis. J Nerv Ment Dis. 2003;191(1): 45-49.

8. Lecomte T, Cyr M, Lesage AD, Wilde J, Leclerc C, Ricard $\mathrm{N}$. Efficacy of a self-esteem module in the empowerment of individuals with schizophrenia. J Nerv Ment Dis. 1999; 187(7):406-13.

9. Hoffmann H, Kupper Z, Kunz B. Hopelessness and its impact on rehabilitation outcome in schizophrenia-an exploratory study. Schizophr Res. 2000;43(2-3):147-58.

10. Lysaker PH, Bell MD, Zito WS, Bioty SM. Social skills at work: deficits and predictors of improvement in schizophrenia. J Nerv Ment Dis. 1995;183(11):688-92. 
11. Zimmerman M, Coryell W, Corenthal C, Wilson S. Dysfunctional attitudes and attribution style in healthy controls and patients with schizophrenia, psychotic depression, and nonpsychotic depression. J Abnorm Psychol. 1986;95(4): 403-5.

12. Lysaker PH, Lancaster RS, Nees MA, Davis LW. Attributional style and symptoms as predictors of social function in schizophrenia. J Rehabil Res Dev. 2004;41(2):225-32.

13. Lysaker PH, France CM. Psychotherapy as an element in supported employment for persons with severe and persistent mental illness. Psychiatry. 1999;62(3):209-21.

14. Stajkovic AD, Luthans F. Self-efficacy and work-related performance: a meta-analysis. Psychol Bul. 1998;124(2): 240-61.

15. Regenold M, Sherman MF, Fenzel M. Getting back to work: self-efficacy as a predictor of employment outcome. Psychiatr Rehabil J. 1999;22(4):361-67.

16. Davis LW, Nees MA, Hunter NL, Lysaker PH. Hopelessness as a predictor of work functioning among patients with schizophrenia. Psychiatr Serv. 2004;55(4):434-36.

17. Van Dongen CJ. Self-esteem among persons with severe mental illness. Issues Ment Health Nurs. 1998;19(1):29-40.

18. Lysaker PH, Clements CA, Wright DE, Evans J, Marks KA. Neurocognitive correlates of helplessness, hopelessness, and well-being in schizophrenia. J Nerv Ment Dis. 2001;189(7):457-62.

19. Brekke JS, Long JD. Community-based psychosocial rehabilitation and prospective change in functional, clinical, and subjective experience variables in schizophrenia. Schizophr Bull. 2000;26(3):667-80.

20. Brekke JS, Levin S, Wolkon GH, Sobel E, Slade E. Psychosocial functioning and subjective experience in schizophrenia. Schizophr Bull. 1993;19(3):599-608.

21. Bond GR, Drake RE, Mueser KT, Becker DR. An update on supported employment for people with severe mental illness. Psychiatr Serv. 1997;48(3):335-46.

22. Davis LW, Lysaker PH, Lancaster RS, Bryson GJ, Bell MD. The Indianapolis Vocational Intervention Program: a cognitive behavioral approach to addressing rehabilitation issues in schizophrenia. J Rehabil Res Dev. 2005;42(1):35-46.

23. First MB, Spitzer RL, Gibbon M, Williams JBW. Structured Clinical Interview for DSM-IV ${ }^{\circledR}$ Axis I Disorders (SCID-I). Arlington (VA): American Psychiatric Publishing, Inc; 1994.

24. Drury V, Birchwood M, Cochrane R, Macmillan F. Cognitive therapy and recovery from acute psychosis: a controlled trial. Br J Psychiatry. 1996;169(5):593-607.

25. Haddock G, Tarrier N, Spaulding W, Yusupoff L, Kinney C, McCarthy E. Individual cognitive-behavior therapy in the treatment of hallucinations and delusions: a review. Clin Psych Rev. 1998;18(7):821-38.
26. Sensky T, Turkington D, Kingdon D, Scott JL, Scott J, Siddle R, O’Carroll M, Barnes TR. A randomized controlled trial of cognitive-behavioral therapy for persistent symptoms in schizophrenia resistant to medication. Arch Gen Psychiatry. 2000;57(2):165-72.

27. Tarrier N, Kinney C, McCarthy E, Wittkowski A, Yusupoff L, Gledhill A, Morris J, Humphreys L. Are some types of psychotic symptoms more responsive to cognitive-behaviour therapy? Behav Cogn Psychother. 2001;29(1):45-55.

28. Kingdon DG, Turkington D. The use of cognitive behavior therapy with a normalizing rationale in schizophrenia. Preliminary report. J Nerv Ment Dis. 1991;179(4):207-11.

29. Rosenheck R, Seibyl CL. Participation and outcome in a residential treatment and work therapy program for addictive disorders: the effects of race. Am J Psychiatry. 1998; 155(8):1029-34.

30. Beck AT, Weisman A, Lester D, Trexler L. The measurement of pessimism: the hopelessness scale. J Consult Clin Psychol. 1974:42(6):861-65.

31. Haatainen KM, Tanskanen A, Kylma J, Antikanien R, Hintikka J, Honkalampi K, Koivumaa-Honkanen H, Viinamaki H. Life events are important in the course of hopelessness-a 2-year follow-up study in a general population. Soc Psychiatry Psychiatr Epidemiol. 2003;38(8):436-41.

32. McClain CS, Rosenfeld B, Breitbart W. Effects of spiritual well-being on end-of-life despair in terminally-ill cancer patients. Lancet. 2003;361(9369):1603-7.

33. Steed L. Further validity and reliability evidence for Beck Hopelessness Scale scores in a nonclinical sample. Educ Psychol Meas. 2001;61(2):303-16.

34. Beck AT, Steer RA. Beck hopelessness scale manual. San Antonio (TX): The Psychological Corporation; 1988.

35. Rosenberg M. Society and the adolescent self-image. Princeton (NJ): Princeton University Press; 1965.

36. Wykes T, Reeder C, Corner J, Williams C, Everitt B. Effects of neurocognitive remediation on executive processing in patients with schizophrenia. Schizophr Bull. 1993;25(2): 291-306.

37. Torrey WC, Mueser KT, McHugo GH, Drake RE. Selfesteem as an outcome measure in studies of vocational rehabilitation for adults with severe mental illness. Psychiatr Serv. 2000;51(2):229-33.

38. Kay S, Fiszbein A, Opler L. The positive and negative syndrome scale for schizophrenia. Schizophr Bull. 1987;13(2): 261-76.

39. Bell MD, Lysaker PH, Beam-Goulet JL, Mistein RM, Lindenmeyer JP. Five-component model of schizophrenia: assessing the factorial invariance of the positive and negative syndrome scale. Psychiatry Res. 1994;52(3):295-303. 
40. Bryson G, Bell MD, Lysaker P, Zito W. The work behavior inventory: a scale for the assessment of work behavior for people with severe mental illness. Psychiatr Rehabil J. 1997; 20(4):48-54.
41. Bryson GJ, Bell MD. Initial and final work performance in schizophrenia: cognitive and symptom predictors. J Nerv Ment Dis. 2003;191(2):87-92.

Submitted for publication December 10, 2004. Accepted in revised form April 21, 2005. 